Paper number EU-SP0232

\title{
Advantage of cooperative traffic light control algorithms
}

\author{
Robbin Blokpoel ${ }^{1}$, Wolfgang Niebel ${ }^{2}$ \\ 1. Research department, Dynniq, The Netherlands, \\ robbin.blokpoel@dynniq.com, +31334541731 \\ 2. Institute of Transportation Systems, German Aerospace Center, Germany, \\ Wolfgang.Niebel@dlr.de
}

\begin{abstract}
Contemporary traffic light control (TLC) systems rely on sensors for detection of traffic which are costly in purchase, installation and maintenance. Emerging cooperative technology (C-ITS) offers an attractive alternative where only one road-side unit (RSU) per intersection is required, instead of several infrastructure sensors per lane. However, studies showed that traffic control with cooperative detection requires a penetration rate of at least $20 \%$ to function effectively. To show the potential of cooperative traffic control, this paper presents three algorithms: (a) the SWARM control algorithm, which is designed to work with very low penetration rates, (b) an extension to the adaptive control algorithm, ImFlow, which uses cooperative data for enhanced queue modelling, and (c) an ImFlow extension to stabilize green planning to enable Green Light Optimal Speed Advice. The results from micro-simulation show a 7.8\% improvement for stops and delay time over traditional adaptive control for Swarm, and $14.9 \%$ for Cooperative ImFlow. Adding planning stabilization reduced the average perceived change for end users from $9.0 \%$ to $2.3 \%$, without performance loss for the overall traffic flow. This shows the large potential of cooperative traffic control.
\end{abstract}

\section{Introduction}

Without traffic monitoring, i.e. without 'knowing' the traffic positions, only fixed time control on intersections is possible. Various plans for different time-of-day, day-of-the-week, or special events can be designed and optimized offline [1], but it is not possible to deal with day-to-day variations. For this purpose, several dynamic control methods exist, each with their own detection requirements. One of them is online plan selection, which selects a fixed time plan based on the current traffic conditions. This method requires at least a few detectors at important locations to determine the traffic state. Another method is vehicle-actuated control in which each signal group needs a detector to determine whether a phase can be cut off or needs extension. Furthermore, adaptive control goes one step further by building a model of the traffic approaching each signal group. Many different adaptive traffic controllers on the market have their own detection requirements, but generally require at least one upstream entry detector per approach. Examples of adaptive control are SCOOT [2], Utopia [3] and ImFlow [4].

Part of the work in this paper builds on ImFlow, which uses an optimizer to test signal plans on the model of the traffic approaching the individual signal groups. These plans are evaluated and selected, based on a cost function which is configured according to the policies specified by the road operator. Public transport priority, bicycle wait time reduction and 
reduction of emissions are examples of such policies that can be implemented by the control algorithm.

For traditional traffic monitoring techniques, installation and maintenance are expensive [5] and may require temporal lane closure. Furthermore, these techniques are susceptible to breakdown and may not work under certain conditions, like fog or heavy snow, reducing the reliability of the system. Therefore, emerging cooperative techniques like vehicle-to-infrastructure (V2I) communication [6] can open new channels for delivering information. However, studies showed that cooperative traffic control systems require large penetration rates of equipped vehicles above $20 \%$ in order to assure their functionality [7], [8], making the first steps towards their deployment unattractive. In order to overcome this hurdle, the COLOMBO project supplied a set of modern, self-organizing traffic management algorithms being applicable even at OBU penetration rates below 10\%, ensuring their usability from the very first deployment days.

Earlier publications dealt with specific applications of cooperative data for traffic control. In [9] this is applied to the adaptive traffic control algorithm ImFlow, but only on a simplified approach without separate signal groups for different turn directions. The work in [10] has separate signal groups, but only considers $100 \%$ penetration. The main traffic control strategy of the COLOMBO project, Swarm, is presented in [11]. This algorithm is a mixture of self-learning plan selection and actuated control, but based on cooperative data and suitable for all penetration rates. All previous work from [9,10,11] was micro-simulated on different synthetic or simplified real-world networks. In order to draw good conclusions about the effects of cooperative data on traffic control, it is required to test both Swarm and the integration of ImFlow with cooperative data on the same network. This network should also be a realistic real-world situation that can indicate how well the systems would perform on other deployment locations. Therefore, in this paper both algorithms are tested in a real-world simulation scenario in the city of Monza. The main corridor under investigation has challenging traffic conditions with daily congestions occurring in the rush hour. The network consists of three consecutive controlled intersections, of which one is complex and two are simple.

As the word cooperative already indicates, both parties cooperate. Therefore, the flow of information from the traffic light controller to the vehicles is also an important aspect. This information can be shared through MAP (Map Data) and SPaT (Signal Phase and Timing) messages [12] and vehicles can use it for Green Light Optimal Speed Advice (GLOSA). This system was shown in $[13,14]$ to have a potential $\mathrm{CO}_{2}$ reduction of up to $7.0 \%$. However, such applications are only possible with a predictable traffic control algorithm. Fluctuations in the prediction when the light will turn green directly lead to fluctuations in the speed advice. This leads to low driver acceptance and reduces the effectiveness of the system. In order to control the stability of the time to green prediction, a trade-off between predictability and flexibility has to be made. Less flexibility generally results in a higher average delay, which is also undesirable.

The feasibility of GLOSA in the control algorithm is, therefore, an important aspect of cooperative traffic control. This is the second objective of the work in this paper and it is done by evaluating if a prediction can be made available and if it is stable. Additionally, the performance of average delay time cannot deteriorate significantly. For this topic, the focus is on the control algorithm itself, while vehicle behaviour modelling with respect to speed advice is considered out of scope. The micro-simulation will still consider all other aspect of 
vehicle behaviour. The research on GLOSA feasibility is part of the MAVEN project [15], which aims at exploiting the possibilities of autonomous vehicles in urban traffic. The work is also part of the XCycle project [16], where it is used for a dynamic bicycle green wave with speed advice. A further reduction of $\mathrm{CO}_{2}$ emissions with GLOSA is one of the applications for this project and a deep understanding of the trade-off between predictability and flexibility is vital.

The paper first introduces the importance of detection for traffic control and how detection is used for both ImFlow and Swarm. This is followed by a description of the control principles behind the algorithms. The simulation scenario, using the open source simulator SUMO [17], is described afterwards. The main focus of the paper is on the results, with a deep analysis of the observed differences between ImFlow, Swarm, and cooperative ImFlow. These main findings are summarized in the conclusion section.

\section{Detection for traffic control}

As described in the introduction, only fixed time control is possible without detection. In order to get a better understanding of the functions of detection, consider Figure 1:

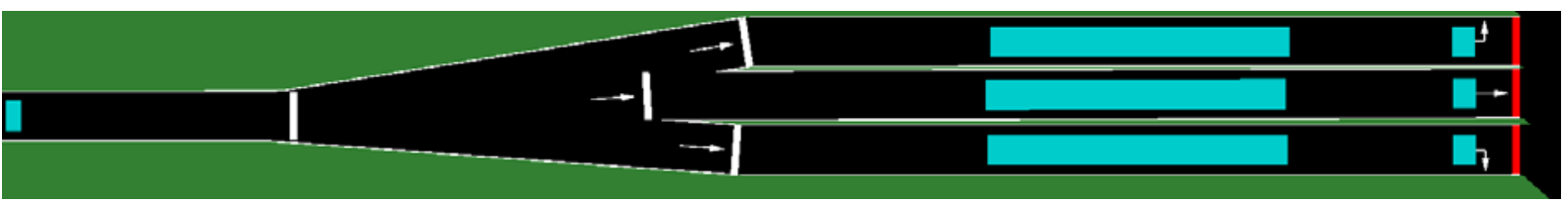

Figure 1: Common detection field for traffic control

Close to the stop line there is a detector, indicated by a blue rectangle. For actuated control, this detector checks if there is demand for a signal group. If this is not the case, the green phase can be skipped. The large detector located at approximately $20 \mathrm{~m}$ from the stop line is the extension detector. The vehicle actuated controller uses this large inductive loop to detect whether the flow of a signal group has ended (therefore, it is also known as gap detector).

For adaptive control, the stop line detector is also used to track the waiting time of vehicles. This may allow for longer cycle times when the waiting vehicle arrives later than the start of red. The entry loop is located further away from the intersection. This is the leftmost detector in Figure 1. It is normally only used for adaptive control and can be up to 1 kilometer upstream of the intersection. Commonly, the function of this loop may be taken over by loops of an upstream intersection. The purpose is to measure approaching vehicles.

One of the main shortcomings of actuated control occurs when consecutive vehicles leave a gap that is slightly larger than the size of the loop. This is illustrated by the situation in Figure 2. The figure uses actuated control and, in both cases, the signal group from the east was green, but was switched to amber because of a gap in the platoon. For an adaptive controller the decision whether to extend the green phase or not is based on how large this gap is and how many vehicles are behind the gap. In a situation where there are many vehicles waiting at the other signal groups, it is probably not worth extending the green for a single vehicle if there is a large gap. In case there is a platoon of 10 cars after this gap, the situation is different. In Figure 2, two different situations are shown. The situation at the top would require further extension of the green, while, in the situation at the bottom, the algorithm should have switched the light to green for the north-south direction earlier to create a green wave for the approaching vehicles. This is where adaptive traffic control can make a better decision thanks to the knowledge acquired by the entry detector. 
Lastly, exit detection is sometimes used to detect downstream congestion. In case of actuated control, the signal groups leading to the congestion will be switched to red to prevent spillback onto the intersection area. In case of adaptive control, such a detector is not required. The queue model of the downstream intersection can be used to detect spillback.
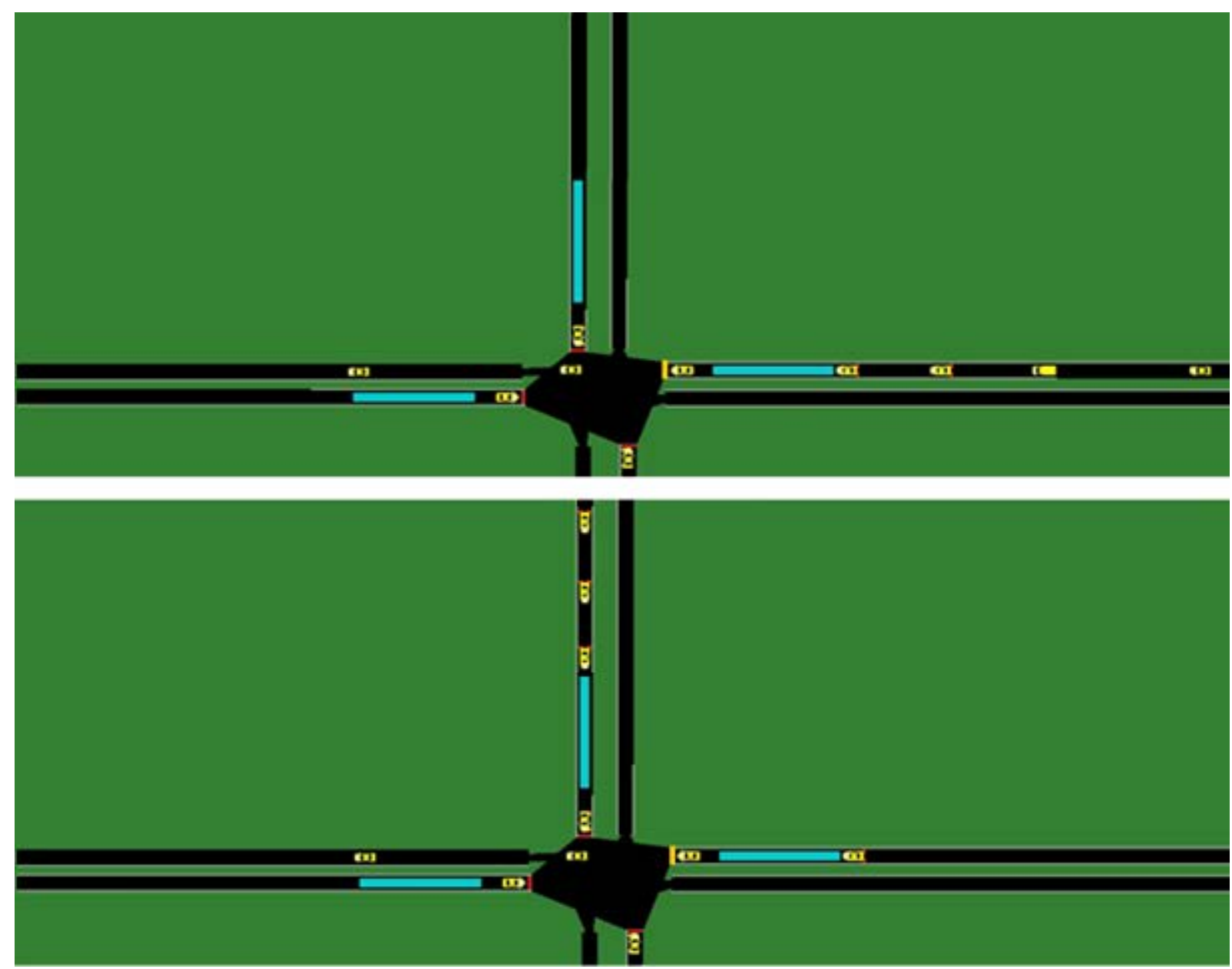

Figure 2: Two situations that appear the same to a vehicle actuated controller, but require a different emission optimal decision

When comparing these functions to the possibilities of cooperative detection at various penetration rates, the data acquisition method should be taken into account. A convenient method to obtain data from cooperative vehicles is to receive their cooperative awareness messages (CAM) with Road Side Units (RSU). These messages are broadcasted with a frequency between 1 and $10 \mathrm{~Hz}$, according to the standard [18], and the speed and position information is used as input for the traffic control algorithms. The vehicle route is missing in the standard, but it is assumed this will be included in the future. After reception of the message, the indicated GPS position of the vehicle is mapped on the road network to determine the distance and travel time to the stop line. With that information, the vehicle can be placed in a queue and arrival model for adaptive control.

In theory, this works for all cooperative penetration rates but, with lower rates, gaps will fall in the data. For example, with a penetration rate of $10 \%, 9$ out of 10 vehicles will not be detected. To compensate for missing data, the COLOMBO project uses an algorithm based on pheromones on both incoming and outgoing lanes, which is calculated as follows: 


$$
\varphi(t)=\beta \varphi(t-1)+\gamma\left(v_{\max }-v_{m e a n}\right)
$$

where $\beta$ is the evaporation coefficient $(0<\beta<1)$, which reduces the amount of pheromone over time. The accumulation coefficient, $\gamma$, depends on the difference between $v_{\text {max }}$, which is the maximum allowed speed on the selected lane, and $v_{\text {mean }}$, which is the measured average speed of the vehicles on that lane. As a function of speed drop, the pheromone is a surrogate measure for the degree of congestion on the lane. The pheromone is used both for plan selection (macro policy) and to evaluate when to switch to the next stage in the control program (micro policy). This means the function of the extension and entry detectors are combined into a large zone detector, spanning from the stop line to $100 \mathrm{~m}$ upstream of the intersection. Pheromone levels on the outgoing links replace the function of exit detectors to prevent spillback.

At the stop line, most control algorithms assume $100 \%$ accurate data to determine whether there is a vehicle waiting or not. Based on that data, the decision to skip a signal group during a cycle is made. When the decision to skip a signal group is made wrongly, a road user may be tempted to violate the red light due to extremely long waiting times. Therefore, the replacement of this detector with cooperative detection to be able to skip signal groups during a cycle would only be possible with $100 \%$ penetration.

\section{Traffic control algorithms}

As described earlier, the cooperative data is used for both the adaptive control algorithm ImFlow and the Swarm algorithm. For adaptive control, the vehicles can simply be injected into the arrival and queue model. In Figure 3, a schematic view of the queue and arrival model is shown:

Distance to stop line [s]

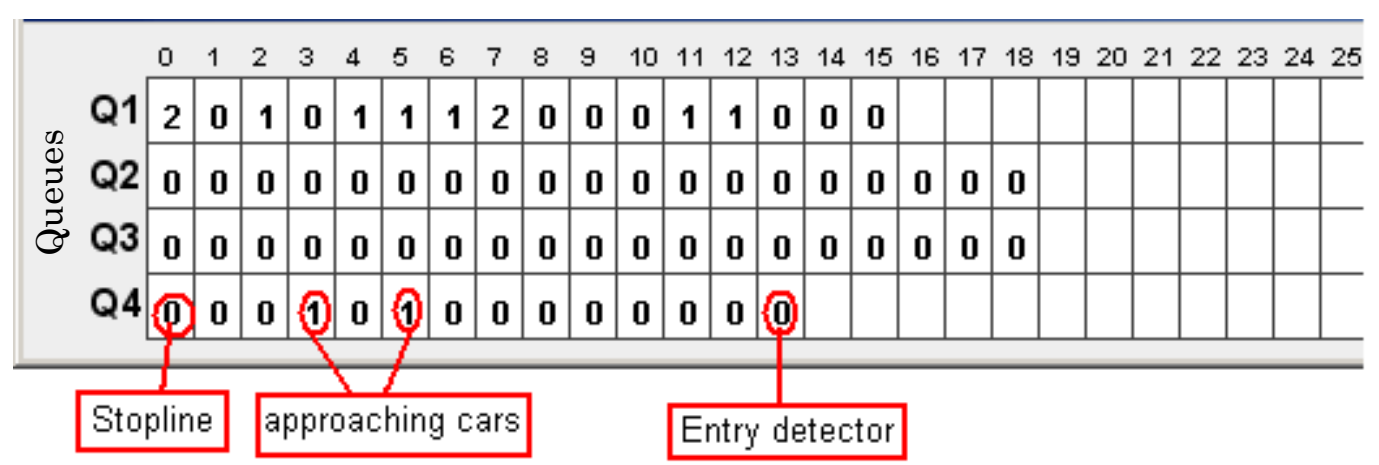

Figure 3: Arrival and queue modelling in adaptive control

Traditionally, the vehicles are injected in the arrival pattern when they pass the entry detector. The x-axis represents the distance to the stop line in travel time. Every second, vehicles in the arrival pattern are moved one field closer to the stop line, which is indicated by the " 0 " column. The queues Q1 to Q4 accumulate at the stop line and discharge with counts from the stop line detector. Cooperative detections can simply overwrite this model. Speed and distance to the stop line can be used to determine the remaining travel time for the arrival pattern. Counting the number of waiting cars determines the current queue length.

ImFlow adaptive traffic control uses a cost function ("impact”) to evaluate signal plans on the planning horizon and executes the plan with the lowest cost. Two cost function parameters are directly affected by changes in the queue estimation or arrival flow pattern: the cost for a 
second of vehicle delay and the cost for a vehicle stop. This can be further explained by the following formula:

$$
C=\sum_{q=0}^{Q} \sum_{t=0}^{T} D W \cdot q(t, p)+S W \cdot s(t, p)
$$

The formula sums over all queues $(Q)$ and in time over the entire planning horizon $(T)$. The weight for vehicle delay and stops is indicated by $D W$ and $S W$ and can be different for each queue. The number of vehicles waiting in the queue $(q)$ and number of vehicles stopping $(s)$ are both a function of the current time step $(t)$ and the control plan $(p)$.

Consider for example Q4 from Figure 3, which has no vehicles in the queue and arrivals at $t=3$ and $t=5$. When this queue is evaluated for 10 seconds of red time, it results in the following costs: the vehicle at $t=3$ stops and has to wait for 7 seconds, the vehicle at $t=5$ stops and has to wait for 5 seconds. When the cost of a stop $(S W)$ is configured as 8 and the delay $(D W)$ as 1 , the total cost for this signal group is 28 seconds $(7+5+8+8)$. When a signal group has a green phase during the planning horizon, the costs stop accumulating for each vehicle as soon as it leaves the queue. It should be noted that when a vehicle arrives during green while there is still a queue, it will incur stop and delay costs. Therefore, the optimizer can try to plan this green phase earlier to allow the vehicle to pass without stopping. With many conflicting signal groups on an intersection, the entire optimization process is complex, since a gain on one signal group may lead to a larger loss on another.

From the aforementioned, it can be understood that accurate information is vital for the controller to make optimal decisions. In practice, the queue length is used to determine the base length of the green phase, while arrivals can be used for green phase extension and the dynamic creation of green waves. Errors in the observation can lead to green phases being too long or too short, causing unnecessary stops.

For the Swarm algorithm, the amount of pheromone, which depends on the intensity of the traffic and whether there is congestion on the outgoing links, is used for the policy selection. This concept is similar to the plan selection algorithm. Once a policy is selected, the controller behaves similarly to an actuated controller, switching the lights according to pheromone levels, with specific rules that depend on the policy. More details on this can be found in [11].

A major difference between the implementation of the Swarm algorithm and ImFlow is that Swarm lacks a stop line detector and is not able to determine if there is demand for a signal group at less than $100 \%$ penetration. Additionally, ImFlow uses a maximum waiting time per signal group of 120 seconds, which can be measured with the stop line detector. For Swarm, each stage has a maximum time, which is required to keep the cycle time limited by the sum of all maximum green times. Effectively, this means ImFlow will be able to use stage times beyond the maximum of Swarm, as long as this does not make any vehicle wait longer than the configured maximum.

\section{Signal state predictability for GLOSA}

With cooperative technology, the information flow from the traffic light controller to the vehicles raises the opportunity to supply a Green Light Optimal Speed Advice (GLOSA). Especially for autonomous vehicles, there is a large potential for $\mathrm{CO}_{2}$ reduction, as they can follow very precise speed advice. However, with such precision, the information in the advice 
should be based on highly accurate data from the traffic light controller. In order to illustrate the importance of this, four fictive speed advice scenarios are depicted in Figure 4.

The first (red) receives no advice at all. This vehicle has to stop, wait for a while and reaccelerate to the desired speed. The second (blue) slows down at the beginning of the time horizon, but it actually slowed down too much due to inaccurate speed advice. Therefore, halfway, the vehicle has to reaccelerate again to a higher speed and finally, after the intersection, accelerate back to the default speed. For the third case (orange), the vehicle doesn't slow down enough and has to slow down further halfway its path to the intersection. The last case (green) has a perfect speed advice at once. Considering this figure, it is clear that the total re-acceleration and $\mathrm{CO}_{2}$ emissions are the largest for the red case and the smallest for the green. The orange and blue cases still have a $\mathrm{CO}_{2}$ reduction, but the changes in advice can be frustrating to a driver if they occur frequently.

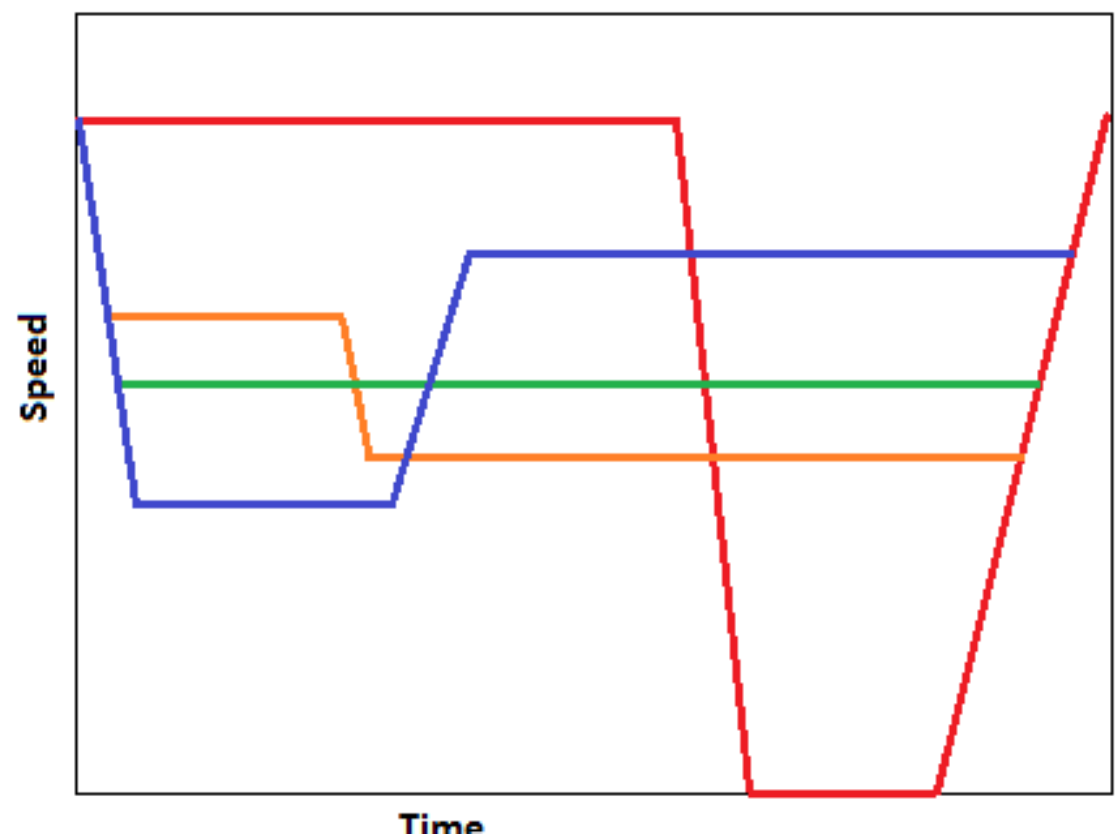

Time

Figure 4: Possible speed advice scenarios

With the effects of non-reliable speed advice demonstrated, it also becomes apparent that the orange and blue cases can have more than a single speed change during an approach towards the intersection. The more these advice changes occur, the smaller the $\mathrm{CO}_{2}$ reduction, up to a point the fuel consumption will even go up when compared to the baseline without speed advice.

When looking at different types of control, the static controller is the most suitable for this cooperative speed advice, considering it is predictable by definition. However, the traffic delays generally increase, as there is no flexibility for changing the control plan according to traffic demand. Even a small variance between different cycles can already cause a queue that will not dissolve when an intersection is at its saturation point.

The actuated controller is at the other extreme. This type of control can switch to the next stage at any point between a configured minimum and maximum green duration. Therefore, a best guess is to use a running average of the last cycles and adjust the green prediction once 
the controller decides to deviate from those values. This causes the prediction of time to green for the next stages to suddenly make a downward jump, in case a previous stage was terminated early. Alternatively, the predicted time stagnates at a certain value, when a previous stage is extended beyond the running average duration. Such a stagnation means the controller gives a predicted time to green of for example 50 seconds, but it stays at 50 seconds until the current green phase is not further extended. The road user will suddenly see its speed advice increase with a cutoff of a previous green phase, while the speed slowly decreases when the prediction stagnates. A traffic engineer can configure the minimum and maximum green times really close to each other to increase the reliability of the speed advice. This reduces the flexibility and, therefore, will have a negative impact on the average delay time of vehicles passing the intersection.

The SWARM strategy can best be compared with an actuated controller. The actuation is not based on the occupancy of detectors, but on the comparison of pheromone levels. The fact that these pheromones increase and decrease gradually means it should be possible to get a more reliable prediction by monitoring the derivative of them.

Looking at the adaptive controller, there is a possibility to add an additional element to the cost function used for optimization. As already explained stops and delay time are standard elements, but plan stabilization is not. A patent for adding an element to the cost function that depends on the time to green prediction change compared to the previous time step was filed in [19]. This should make the control algorithm more suitable for GLOSA, without deteriorating the average traffic delay significantly. The implementation of this cost function is further explained in the following formula:

$$
\begin{gathered}
C=\frac{S B W \cdot d^{2}}{T T G_{t-1}} \\
d=T T G_{t-1}-T T G_{t}-T
\end{gathered}
$$

The configured weight for stability ( $S B W$ ) is comparable to the weights for delay and stops of formula (2). The deviation (d) is calculated using the difference between the time to green (TTG) of two consecutive time steps. The time period of a time step (T) is used for the expected decrease of the TTG. The cost is quadratic with respect to the deviation because higher deviations are increasingly worse for the driver acceptance of a speed advice. Furthermore, the cost is inversely proportional to the TTG of the last time step. This is because the closer to green, the more impact a change in the plan has.

An added advantage of a configurable cost function element is that it can be activated on a per signal group basis. The through direction of the main road generally has the largest amount of traffic and, therefore, the highest benefit of GLOSA and plan stabilization. Other directions can be more flexible because they don't require a cost for plan stability. This way the controller is able to combine the best of two worlds: stability for the main direction and flexibility for the others.

In order to evaluate the users perception of a speed advice in simulation, it is not possible to do questionnaires with virtual drivers. It is, however, clear that large changes between consecutive TTG predictions are not desirable. A measure like the mean square error (mse) of the prediction gives a clear indication about the quality of the prediction. On the other hand, it is important to consider the consequences of the moment the change occurs as well. A 5 
second change in the time to green has a much smaller impact when the TTG is 50 seconds than when it is only 5 seconds. The first results in a $10 \%$ change of the speed advice, while the latter means it is cut in half. Therefore, the time to green will be evaluated according to a "perceived change":

$$
p c=\sum_{t-1}^{7200 \frac{T T G_{t-1}-T T G_{t}}{\min _{>0}\left(T T G_{t-1}, T T G_{t}\right)} 100 \%} / \sum_{t=1}^{7200} i f\left(T T G_{t}\right)=\left\{\begin{array}{l}
0, T T G>60 \\
1, T T G \leq 60
\end{array}\right.
$$

The perceived change is averaged over the total amount of cases when a prediction is relevant. Considering the maximum distance between intersections is $600 \mathrm{~m}$ and $30 \mathrm{~km} / \mathrm{h}$ is a good lower limit for a speed advice, the 60 second limit would be a good rule of thumb for advice relevance.

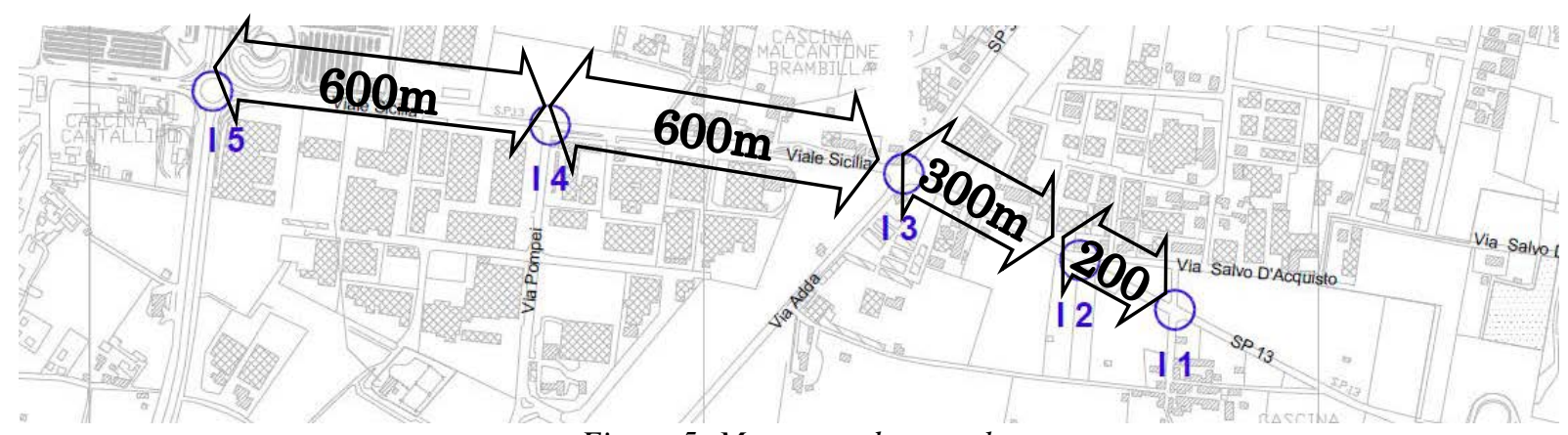

Figure 5: Monza road network

\section{Monza simulation scenarios}

The micro-simulation scenario consists of a SUMO 0.24.0 network of the city of Monza, Italy, shown in Figure 5. The demand of the scenario is based on traffic counts at the morning rush hour between 7:00 and 9:00 a.m. in 15 minute intervals [20]. A Poisson distribution is applied to the demand for a realistic fluctuation in the arrival pattern. The simulation was also calibrated with modifications to the intersection layout to model the uncontrolled intersections more accurately. The model encompasses a stretch of 3 signalized intersections (I1, I3, I4) on the south-western Viale Sicilia. I2 is a yielding junction, and I5 is a complex roundabout which serves only as origin/destination.

Figure 6 shows the lanes and signal groups on the controlled intersections in more detail. From this, it can be concluded the network has many different aspects that can occur in urban environments. I1 and I4 are simple intersections with one lane allowing all possible turn movements. I3 has 2 lanes for the ongoing traffic on both roads and separate lanes for the left turn. The right turn in this intersection is either with an uncontrolled curve bypassing the intersection or shared with the rightmost ongoing traffic lane. This layout is generally a problem for traditional traffic control, as turning percentages have to be estimated to assign arriving traffic to one of the lanes.

Pedestrians are present only on the east side of I1 and all sides of I3. They get green in the same stage as the parallel car movements. A challenging situation in this network is the fact that I3 has double lanes while I1 and I4 only have a single lane. Therefore, vehicles have to merge in approximately $150 \mathrm{~m}$ after the intersection. This can easily cause congestion on an outgoing link. 


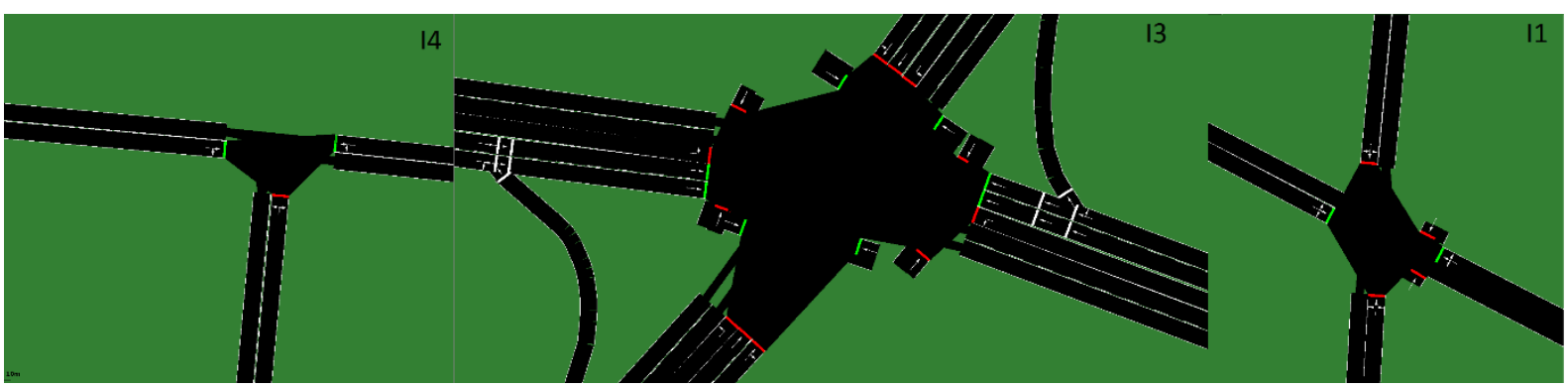

Figure 6: Monza intersection layout

The scenarios are simulated at various penetration rates and with a traditional adaptive controller as base line. Each scenario is simulated 30 times with a different random seed and results are averaged afterwards. Results are acquired per signal head and then averaged for the entire network.

For the plan stability two scenarios of the same network of Figure 6 are simulated. However, this network has problems with uncontrolled intersections, which on rare occasions block a traffic stream. Normally, an adaptive controller detects that and switches immediately to prevent spillback. For GLOSA, such abrupt and unexpected spillback events are very disruptive and, therefore, the right of way rules on those intersections have been removed in order to prevent this situation. As such, the GLOSA simulation scenario cannot be compared to the other scenarios and will be considered in a separate sections of results.

\section{Results}

During the simulations, it became clear that it was very challenging for the adaptive algorithm to estimate the turning percentages on intersection I3. Cycle-by-cycle fluctuations resulted in many inefficient control decisions, as the queues were not estimated correctly. Regular intersections do not have this problem but, when it occurs, the traffic engineer generally constrains the adaptive algorithm further to mitigate the effects of inaccurate queue estimations. In this research, the algorithm was not constrained so the differences between swarm, adaptive and cooperative adaptive control would be more visible.

The overall results are summarized in Table 1. It should be noted that delay is averaged per vehicle and per signal group. A vehicle passing all intersections with a delay time of 10, 20 and 30 seconds will be showing in these statistics with an average delay of 20 seconds. Stops are averaged in a similar manner per signal group and per vehicle. The impact as aggregated measure consisting of delay time + stops $* 8$ was already described previously. 
Table 1: Overall results for different control strategies

\begin{tabular}{|l|r|r|r|r|}
\hline Scenario & Throughput [veh/2h] & Impact [s] & Delay [s] & \multicolumn{1}{l|}{ Stops } \\
\hline adaptive & 673754 & 99.50 & 73.49 & 3.25 \\
\hline swarm 1\% & 672795 & 101.13 & 70.04 & 3.89 \\
\hline swarm 2.5\% & 669072 & 99.57 & 69.06 & 3.81 \\
\hline swarm 5\% & 672842 & 98.26 & 68.25 & 3.75 \\
\hline swarm 10\% & 672651 & 94.82 & 65.79 & 3.63 \\
\hline swarm 25\% & 674683 & 93.46 & 64.88 & 3.57 \\
\hline swarm 50\% & 678343 & 92.26 & 64.75 & 3.44 \\
\hline swarm 100\% & 673050 & 93.33 & 65.10 & 3.53 \\
\hline adaptive CAM & 682350 & 86.62 & 63.37 & 2.91 \\
\hline $100 \%$ & & & & \\
\hline
\end{tabular}

The throughput is mostly a control measure. This is to prevent a solution that gives a lot of green to a subset of signal groups while only letting one vehicle per cycle pass for the others. This way, the throughput is so low that the impact on the average delay is insignificant. In this case, the difference between the maximum and minimum throughput is less than $2 \%$, meaning that all solutions are acceptable. More throughput is generally better, but the differences in impact are more apparent. For the impact, Swarm control is better than adaptive at penetration rates higher than 2.5\%, which can be explained by the challenging situation for queue estimations. These are based on a measured running average of the turning percentages. The cooperative data may not be very complete, but the turning ratios are known from the route information in the CAM, instead of estimated. Also it can be noted that the biggest performance gain is from 5 to $10 \%$ penetration. Therefore, reaching $10 \%$ can be considered an important milestone for the introduction of swarm based traffic control. It is interesting to see the worsening impact from $50 \%$ to $100 \%$. This is an effect that is observed more often in algorithms dealing with incomplete data. It can be considered a direction for future improvement to enable the algorithm to deal with complete data as well. In conclusion, Swarm has a 7.8\% improvement for the impact over traditional adaptive control. Combining the adaptive control algorithm ImFlow with full knowledge of the CAM results in an even larger improvement of $14.9 \%$.

Another interesting observation is the difference in stops and delay. ImFlow uses both stops and delay in its cost function, while SWARM only optimizes for delay time. This results in ImFlow always being better for stops, but at the cost of delay time. When looking over the 2 hours of simulation the following can be observed in Figure 7: 


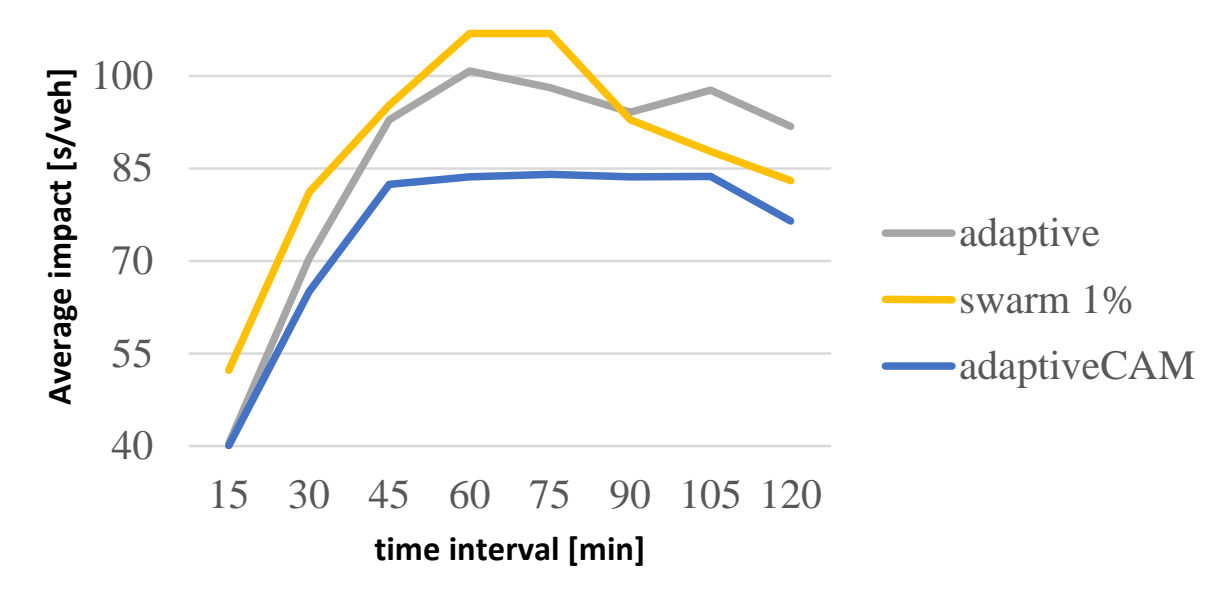

Figure 7: Impact in [s] averaged over 15 minute intervals

In this figure, it is interesting to observe that Swarm has more variation in the impact over time, but checking the variance of the impact on a per vehicle basis within the individual time intervals did not reveal significant differences. Additionally, the results are based on the average of 30 simulation runs, which entails a high confidence. In conclusion, all control strategies can be considered stable, but Swarm performs relatively better in the last 30 minutes.

So far all results were presented over the entire network. As described earlier, the scenario has various different situations. Considering them separately can be of interest to evaluate where cooperative data really makes a difference. Therefore, Table 2 shows an overview of the most significant subsets of the network:

Table 2: Results for various subsections of the network

\begin{tabular}{|l|r|r|r|}
\hline Scenario & \multicolumn{1}{|c|}{ Impact [s] } & \multicolumn{1}{c|}{ Delay [s] } & \multicolumn{1}{l|}{ Stops } \\
\hline adaptive CAM 100\% (I4) & 139,922 & 90,833 & 6,136 \\
\hline adaptive (I4) & 123,875 & 78,610 & 5,658 \\
\hline swarm 50\% (I4) & 124,871 & 78,814 & 5,757 \\
\hline adaptive CAM 100\% (I3) & 76,720 & 62,276 & 1,805 \\
\hline adaptive (I3) & 109,118 & 88,224 & 2,611 \\
\hline swarm 50\% (I3) & 101,279 & 77,159 & 3,015 \\
\hline adaptive CAM 100\% (pedestrians) & 53,430 & 45,019 & 1,051 \\
\hline adaptive (pedestrians) & 55,527 & 47,040 & 1,060 \\
\hline swarm 50\% (pedestrians) & 58,374 & 50,284 & 1,011 \\
\hline
\end{tabular}

The results for pedestrians (included in both algorithms) show that ImFlow prioritizes pedestrians slightly, compared to normal traffic. It is interesting to see that the overall best control strategy actually performs worse on the simple intersection I4. This is caused by the fact that I3 causes more spillback onto I4 for the west-east direction with this control strategy. For the overall network, this is better and it can also be seen in Table 1, where adaptive CAM has the highest throughput and the lowest impact. The east side has less space to store long queues and was therefore prioritized. 
A closer look at the total traffic volumes per signal group also revealed that the scenario was slightly oversaturated, as the east-west throughput did increase significantly with $10 \%$ for the adaptive CAM scenario when compared to 50\% penetration rate for Swarm. This means the impact improvement will be larger if the traffic demand is reduced to the level that can be served by the other solutions.

Comparing the results with the simple single lane cross scenario in [11] shows that cooperative data excels at more complex intersections. In [11] Swarm was able to improve only approximately $2 \%$ over actuated control. While in this complex scenario a more sophisticated controller than actuated control was used and still the performance gain was larger. This can be explained by the difficulty of estimating the turning percentages. When connecting these findings to the detailed results of the Monza scenario in this research, it can be concluded that the most complex intersection benefits the most from usage of cooperative detection. Interestingly, the neighboring intersections can suffer from more efficient traffic handling, attenuating the gains when looking at an overall network level. The pedestrians benefit from overall more efficient traffic control. Their delay time and stops are not influenced by spillback or platooning effects, but they benefit from the possibility of running shorter cycles.

\section{Predictability results}

To investigate the predictability of the control algorithm the time to green was logged during the entire two-hour simulation runs. With post-processing, the actual time to green can be calculated by tracing back from the moment the light turned green. This resulted in a good overview of the functional performance of the signal plan stabilization applied to Adaptive CAM, as is shown in Figure 8:
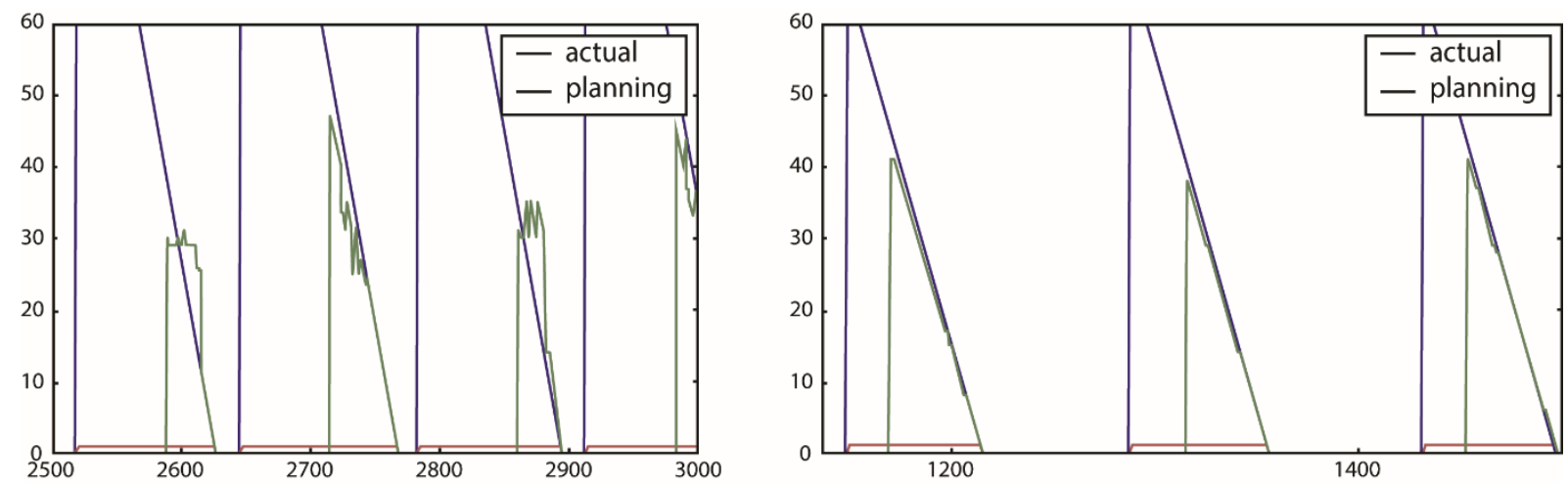

Figure 8: Actual vs. predicted planning for the baseline (left) and stabilized (right) scenario's. The x-axis represents time and the y-axis the (predicted) time to green.

As can be seen in the figure the non-stabilized control strategy results in many jumps for the time to green. This does not necessarily result in a large mean square error (MSE) when compared to the reference time to green. For example, a prediction that jumps from an error of -5 seconds, to +5 and back to -5 , will result in the same MSE as a prediction that stays constant at -5 seconds. However, a user who receives a speed advice based on this data will be confused seeing two speed changes in a short period of time. This is where the perceived change, as introduced in equation (2), plays an important role for evaluation. The stabilized plan on the right of the figure shows a much more stable prediction. Small changes are still occurring and are based on a decision by the cost function. Such decision takes into 
consideration whether this small change provides enough gain to the other traffic, compared to the impact on GLOSA systems.

Table 3: Stability analysis results

\begin{tabular}{|l|l|l|l|l|l|l|}
\hline Scenario & $\begin{array}{l}\text { Impact[s] } \\
\text { average }\end{array}$ & $\begin{array}{l}\text { Impact[s] } \\
\text { std. }\end{array}$ & $\begin{array}{l}\left.\text { MSE[s }{ }^{2}\right] \\
\text { average }\end{array}$ & $\begin{array}{l}\left.\text { MSE[s }{ }^{2}\right] \\
\text { std. }\end{array}$ & $\begin{array}{l}\text { pc[\%] } \\
\text { average }\end{array}$ & $\begin{array}{l}\text { pc[\%] } \\
\text { std. }\end{array}$ \\
\hline Baseline & 49.97 & 4.32 & 104.89 & 46.80 & 9.02 & 4.54 \\
\hline Stabilized & 47.22 & 4.63 & 67.67 & 39.73 & 2.34 & 0.64 \\
\hline Baseline 60\% & 20.22 & 0.59 & 4.74 & 1.62 & 1.90 & 0.22 \\
\hline Stabilized 60\% & 20.45 & 0.49 & 5.54 & 1.66 & 1.77 & 0.08 \\
\hline
\end{tabular}

There is $60 \%$ of the traffic volume in the scenarios identified with "60\%", which is a general rule of thumb to simulate the off peak traffic during daytime. For the peak hour, the impact actually decreases by adding the stabilization, but this result only has a confidence of $81 \%$ according to the student's t-test. For the off peak, the non-stabilized configuration performed better with a very low confidence of $64 \%$. From this, it can be concluded that the overall impact on all traffic does not change significantly. When looking at the MSE of the prediction, there is an improvement with 93\% confidence, while the less challenging off-peak situation has no significant change. As already explained before, the MSE is not the right tool to evaluate this particular system. The perceived change evaluates the users experience directly and has a very large reduction during the peak hour (confidence $>99.99 \%$ ) and a small reduction (confidence $>90 \%$ ) for off peak.

Most important here is to define which level of perceived change is acceptable for GLOSA and ensure this level is guaranteed under normal operation conditions. The system should be configured in a way that a minimum performance of GLOSA is guaranteed. During the peak hour, where the optimization is less stable, the cost function stabilization has a higher relevance. The system is less active during off-peak.

\section{Conclusion}

Previous research with Swarm has shown a large potential on simple intersections compared with actuated control. Similarly, cooperative extensions to ImFlow also showed large improvements in simplified simulation networks. The main research goal of this paper was to compare both systems on a real world simulation scenario. In order to achieve this, both control algorithms were tested on a complex and saturated simulation network in the city of Monza, Italy.

With a 7.8\% improvement over traditional adaptive control for Swarm and 14.9\% for ImFlow extended to support CAM, the results exceeded the expectations for complex networks. It could be concluded that the larger benefits at complex networks, compared to simple networks, were due to difficulty of estimating turning percentages with traditional detection. The only drawback of the proposed systems is their reliance on destination or future path indications in the CAM messages. Although this is a clear requirement for future versions of the CAM message, the current standard does not support this yet.

Planning stabilization by means of adding another objective to an adaptive control algorithm shows a large reduction of perceived change for GLOSA users. During the challenging peak hour, the perceived change reduced from an average $9.0 \%$ to $2.3 \%$. Adding the objective to the optimization did not result in significant overall performance loss. 
This paper has shown the large potential of cooperative data from CAM messages for traffic control. The Swarm algorithm can be seen as the cooperative successor of actuated traffic control, while ImFlow can be considered ready for a cooperative extension for even better adaptive control and GLOSA support when the penetration rate of C-ITS on the road starts to rise.

\section{Acknowledgements}

The work on the SWARM algorithm and cooperative detection was part of the COLOMBO project and therefore the authors want to thank the European Commission for co-funding the COLOMBO project under the grant number 318622. The work on planning stabilization was part of the MAVEN and XCycle projects. The authors want to thank the European Commission for co-funding the MAVEN project under the grant number 690727 and XCycle under the grant number 635975. As well, the authors want to thank all staff members that contributed to the presented results.

\section{References}

1. Gazis D. C., Potts R. B., The Oversaturated intersection, 2nd Int. Symposium on Theory of Road Traffic Flow, Paris, France, 1963, pp221-237.

2. Hunt, P.B., Robertson, D.I., Bretherton, R.D., Winton, R.D., SCOOT, A Traffic Responsive Method of Coordinating Signals. TRRL Laboratory Report 1014, 1981.

3. Mauro, V., Di Taranto, C., UTOPIA, CCCT'89 - AFCET Proceedings, Paris, 1989.

4. Van Vliet, K., Turksma, S., ImFlow: Policy-based adaptive urban traffic control First field experience, ITS Europe, Dublin, Ireland, 2013.

5. U.S. Department of Transport, Traffic detector handbook: Third Edition- Volume II, publication number: FHWA-HRT-06-139, 2006.

6. eCoMove project, Simulation results for validation and impact assessment, Deliverable D5.10 (restricted), 2013.

7. Konstantinos Katsaros, Ralf Kernchen, Mehrdad Dianati, David Rieck, Performance study of a Green Light Optimized Speed Advisory (GLOSA) Application Using an Integrated Cooperative ITS Simulation Platform, IEEE, 2011.

8. Koenders, E., in 't Velt, R., Cooperative technology deployed, ITS Europe, Lyon, France, 2011.

9. Blokpoel, R.J., Kielstra, C., Vreeswijk, J., Data fusion of cooperative data with adaptive traffic control, ITS World, Bordeaux, France, 2015.

10. Blokpoel, R.J., Vreeswijk, J., Sluijsmans, G., New traffic light control strategy based on probe vehicle data, EWGT conference, Delft, The Netherlands, 2015.

11. Krajzewicz, D., et. al., Traffic management based on vehicular communication at low equipment rates, ITS world congress, Bordeaux, France, 2015.

12. SAE standard, J2735, September 2015.

13. Passchier, I., et. al., Influencing driver behavior via in-car speed advice in a field operational test, ITS European congress, Dublin, Ireland, June 2013.

14. Blokpoel, R., Islam, M.F., Vreeswijk, J., Impact analysis of the ecoApproach advice application, ITS European congress, Helsinki, Finland, June 2014. 
15. Blokpoel, R., Lu, M., Pribyl, O, Interoperable architecture between simulation and pilots for cooperative and automated driving, ITS Europe congress, Strassbourgh, France, June 2017.

16. Cassani, M., Pietrantoni, L., XCycle newsletter 2, http://www.xcycle-h2020.eu/NewsAndEvents/Newsletter/2, accessed October 2016.

17. Krajzewicz, D., Bonert, M., Wagner, P., The Open Source Traffic Simulation Package SUMO, RoboCup 2006 Infrastructure Simulation Competition, Germany, Bremen.

18. ETSI standard, Intelligent Transport Systems (ITS); Vehicular Communications; Basic Set of Applications; Part 2: Specification of Cooperative Awareness Basic Service, ETSI EN 302 637-2 V1.3.1, 2014-09.

19. Patent pending under reference number N2018711, 13 ${ }^{\text {th }}$ of April 2017.

20. Centro Studi Traffico / Provincia di Monza e Brianza: Studio per la Definizione e Valutazione del Nuovo Assetto della SP 13 tra Colleoni e Pompei, Milano (IT), 2012. 